\title{
Unanticipated difficult endotracheal intubation due to an undiagnosed pharyngeal web
}

\author{
Jong In Oh, Tae-In Ham, Sung Bae Jeon, Min-Su Kang, and Ho-Yong Shim \\ Department of Anesthesiology and Pain Medicine, National Police Hospital, Seoul, Korea
}

An unexpected difficult airway during the induction of general anesthesia may be challenging for anesthesiologists. We present the case of an unsuspected difficult intubation caused by an undiagnosed pharyngeal web and tracheal stenosis.

An 85-year-old, $40 \mathrm{~kg}, 150 \mathrm{~cm}$ woman was scheduled for a combined operation of cataract and urinary incontinence. In her medical history, she had dementia and Parkinson's disease that were both treated with medication, and her preoperative ECG and chest X-ray showed a complete right bundle branch block and atelectasis in the left lower lobe. In the preanesthetic visit, she was classified as Mallampati I, showing no particular abnormality. General anesthesia was induced with fentanyl 50 $\mu \mathrm{g}$, thiopental sodium $200 \mathrm{mg}$ and rocuronium $40 \mathrm{mg}$. Under direct laryngoscopy (Macintosh curved blade \#3), an oval structure different from the normal shape of the epiglottis and a narrow opening beneath it was seen, and their surrounding tissue presumed to be a pyriform sinus, was observed. After lifting up the epiglottic tip, two lumens were seen through the first opening, which were presumed to be the glottic and esophageal openings. We tried endotracheal intubation using a endotracheal tube with a $6.0 \mathrm{~mm}$ inner diameter (ID) and 8.4 mm outer diameter (OD).

Because the glottis was not clearly observed and the tube could not easily pass the first opening, we reattempted intubation with a reinforced endotracheal tube 5.5 ID (7.5 OD). We could pass the first opening, but the sliding of the tube tip was performed blindly resulting in esophageal intubation. After failure of the third attempt, we recognized high pressure in the breathing bag even though the saturation was maintained at $100 \%$, so we decided to try orotracheal intubation using a fiberoptic laryngoscope (LF-GP, Olympus, Tokyo, Japan). The first opening was confirmed, and as the fiberoptic laryngoscope was progressed slightly further, the glottic and esophageal opening were confirmed below the first opening. The vocal cords were observed as rudimentary and abnormal in shape, however we tried to slide in a reinforced endotracheal tube (5.5 ID), but the area seemed too tight after advancing $2-3 \mathrm{~cm}$. We changed the tube to a smaller diameter and succeeded in fiberoptic intubation using a reinforced endotracheal tube 5.0 ID (6.9 OD). After the operation, the patient recovered without respiratory difficulty.

In the postoperative interview, we found that she had suffered from dysphagia (solid food) since long ago but had not been treated, since the symptoms were not severe. A postoperative computed tomography (CT) showed a pharyngeal web covering the laryngeal part of the pharynx on the posterior pharyngeal wall in the upper part of the hypopharynx, with tracheal stenosis below the C6 level of the cricoid area, and upper esophageal wall thickening that extended to mid-esophagus which was suspected of esophageal cancer, accompanied by an elevation in the piriform sinus and esophageal dilatation. Her rigid laryngoscopic examination (Fig. 1) by otorhinolaryngologists had a similar review as the one that was found during endotracheal intubation and CT findings.

The true glottis and the esophageal opening were observed only after the rigid laryngoscope had passed the first opening that resembled the glottis. The true vocal cord was not developed well, the false vocal cord and pyriform sinus were

Corresponding author: Jong In Oh, M.D., Department of Anesthesiology and Pain Medicine, National Police Hospital, Garakbon-dong, Songpagu, Seoul 138-708, Korea. Tel: 82-2-3400-1323, Fax: 82-2-3400-1270, E-mail: drjj@paran.com

(c) This is an open-access article distributed under the terms of the Creative Commons Attribution Non-Commercial License (http:// creativecommons.org/licenses/by-nc/3.0/), which permits unrestricted non-commercial use, distribution, and reproduction in any medium, provided the original work is properly cited. 

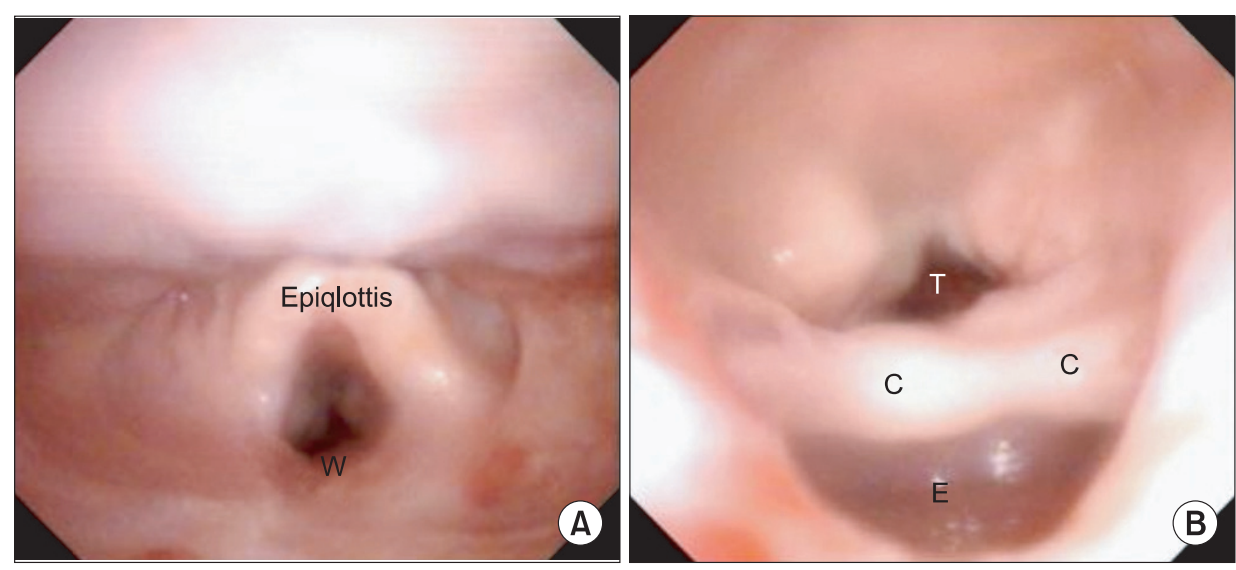

Fig. 1. (A) The rigid laryngoscopic view of the hypopharyngeal web after the operation. It shows a shape similar to the glottis and did not show the esophagus. W: A web formation of the upper part of the hypopharyx. (B) The rigid laryngoscope view as it was advanced through the false lumen. It shows the corniculate cartilages are enlarged and the entrance to esophagus is larger than trachea. T: trachea, C: corniculate cartilage, E: esophagus. very thickened, the cuneiform and the corniculate cartilage were enlarged, and the glottis entrance had become narrow. The patient was given a recommendation to undergo an examination of the pharyngeal membrane for esophageal cancer but the patient refused. After 6 months, she died of esophageal cancer.

In this case, it is uncertain whether the anatomical abnormality of the pharyngeal web and the tracheal stenosis were congenital or acquired. Such a case report is rarely found even among reports in other countries. Considering that the patient had long suffered dysphagia when eating solid food and tracheal stenosis might have been caused by the thickening of the esophageal wall resulting from esophageal cancer, we could not rule out the possibility of Plummer-Vinson syndrome which is associated with pharyngeal web formation and esophageal cancer [1]. The pharyngeal web is not a particular inducer of difficult endotracheal intubation, but in this case, the web was formed on the upper part of the hypopharynx and covered parts of the esophagus and larynx. In addition to these severe anatomical variations, tracheal stenosis had accompanied, and these problems made endotracheal intubation difficult.

According to the difficult airway algorithm suggested by the American Society of Anesthesiologists [2], when the first endotracheal intubation is unsuccessful, the anesthesiologist considers calling for help and determines whether he has to awaken the patient while ventilation continues. If mask ventilation is maintained properly, the anesthesiologist may consider some different methods including the use of different laryngoscope blades, a laryngeal airway mask (LMA), fiberoptic intubation, intubating stylet or tube changer, light wand, retrograde intubation, and blind oral or nasal intubation. As we chose fiberoptic intubation and it was successful, management was appropriate. But in the case of failure, the anesthesiologist may consider awakening the patient, performing an invasive airway access or other feasible options.

In conclusion, it is ideal to predict a difficult airway during the patient's preoperative visit and to prevent difficult airway situations through full preparations, but thanks to recent advanced anesthetic methods and equipment we may be able to overcome difficulties in airway management through careful stepwise handling.

\section{References}

1. Haubrich WS, Schaffner F, Berk JE. Bockus gastroenterology. 5th ed. Philadelphia, Saunders Co. 1994, pp 518-23.

2. Practice guideline for management of the difficult airway: an updated report by the American Society of Anesthesiologists Task Force on Management of the Difficult Airway. Anesthesiology 2003; 98: 1269-77. 